FFP12 2011

Udine 21-23 November 2011

\title{
DIRECTION OF TIME FROM THE VIOLATION OF TIME REVERSAL INVARIANCE
}

\author{
Joan A. Vaccaro, Centre for Quantum Dynamics, Griffith University
}

\begin{abstract}
We show that the violation of time reversal invariance $(T)$ observed in meson decay may have a profound effect on the direction of time. Our starting point is a universe without a presumed direction of time. Time evolution is modeled by taking an equal superposition of steps in both directions of time. This gives rise to multiple paths through time. The presence of $\mathrm{T}$ violating processes is shown to give rise to destructive interference between all paths except for the two that comprise continuously forwards and continuously backwards steps. We show that this leads to a new kind of irreversibility that is quite unlike that found in thermodynamics.
\end{abstract}

\section{Introduction}

The direction of time is typically studied in terms of various arrows of time (Price 1996). Each arrow is associated with some time-asymmetric phenomenon and is oriented to point to the future. Perhaps the best known is the thermodynamic arrow which points in the direction of an increase in the entropy of an isolated system (Eddington 1928). Essentially, an increase in entropy is the effect that results from a fixed direction of time and an incomplete knowledge of a system. The thermodynamic arrow requires the universe to be initially in a low-entropy non-equilibrium state and evolve towards equilibrium. This implies an asymmetry between the past and future boundary conditions. The time asymmetry of other arrows of time can also be attributed to asymmetric temporal boundary conditions. For example, the cosmological arrow points in the direction of the expansion of the universe and so relies on a small universe in the past and a large universe in the future. All such arrows are based on time-symmetric dynamical laws. The one notable exception is the matter-antimatter arrow of time (also called the weak arrow of time) which partially explains the dominance of matter over antimatter in the universe. It arises from the observed violation of charge conjugation and parity inversion invariance (CP) in meson decay due to the weak interaction (Christenson 1964). Under the CPT theorem, CP violation is equivalent to violation of time reversal invariance $(T)$. This means that meson decay is described by a time-asymmetric dynamical law and so the matter-antimatter arrow originates from the time asymmetry of a dynamical law rather than boundary conditions. Of all the arrows of time, it is the only one to have the potential of being the cause of the direction of time, rather than being simply an effect. Indeed $T$ violation in meson decay has recently been shown to have potentially large scale physical effects in a model of the universe in which the direction of time is not predefined (Vaccaro 2011). Here we re-examine the implications of the model for the direction of time.

In Section 2 we briefly review the model that was introduced in (Vaccaro 2011). Next in section 3 we show how a new kind of irreversibility emerges from the model when sufficient $T$ violation processes are present. In section 4 we explore the repercussions of baryogenesis and end with a discussion of the implications for the direction of time in section 5.

\section{Effect of $T$ violation in a universe without a presumed temporal direction}

We want to explore how $T$ violation can give rise to a preferred direction of time. For this we need to model the universe in such a way that it has no predefined direction of time if $\mathrm{T}$ violation processes are absent. We have two choices: the universe does not evolve at all, in which case there is nothing more to be said, or both directions of time have an equal footing. To incorporate the second option, rather than take time steps in a particular direction, time evolution must somehow be in both directions of time. In other words we must allow for the possibility of multiple paths through time. Feynman's sum over paths (Feynman 1948) provides the mathematical framework for dealing with such situations. We let the evolution in one direction of time, which we shall call "forwards" and associate with the positive time direction, be described by

$$
\left|\psi_{F}\right\rangle=U_{F}(\tau)\left|\psi_{0}\right\rangle
$$


FFP12 2011

Udine 21-23 November 2011

and in the other direction of time, which we shall call "backwards" and associate with the negative time direction, by

$$
\left|\psi_{B}\right\rangle=U_{B}(\tau)\left|\psi_{0}\right\rangle,
$$

where $\tau$ represents a time interval and

$$
\begin{aligned}
& U_{F}(\tau)=\exp \left(-i H_{F} \tau\right) \\
& U_{B}(\tau)=T U_{F}(\tau) T^{-1}=\exp \left(i T H_{F} T^{-1} \tau\right)=\exp \left(i H_{B} \tau\right) .
\end{aligned}
$$

Here, $H_{F}$ and $H_{B} \equiv T H_{F} T^{-1}$ are the Hamiltonians for the universe with respect to the forward and backward directions of time, respectively, $T$ is Wigner's time reversal operator (Wigner 1959) and we use units where $\hbar=1$. The Hamiltonian violates time reversal invariance if

$$
H_{B} \neq H_{F} \text {. }
$$

The expressions $\left\langle\phi\left|U_{F}(\tau)\right| \psi_{0}\right\rangle$ and $\left\langle\phi\left|U_{B}(\tau)\right| \psi_{0}\right\rangle$ represent the probability amplitudes for the universe to evolve from the state $\left|\psi_{0}\right\rangle$ to the state $|\phi\rangle$ via two different paths, each of which corresponds to a different direction of time. We have no reason to favor one path over the other so, according to Feynman's sum over paths method, we take the total probability amplitude to evolve from $\left|\psi_{0}\right\rangle$ to $|\phi\rangle$ as the sum $\left\langle\phi\left|U_{F}(\tau)\right| \psi_{0}\right\rangle+\left\langle\phi\left|U_{B}(\tau)\right| \psi_{0}\right\rangle=\left\langle\phi\left|U_{F}(\tau)+U_{B}(\tau)\right| \psi_{0}\right\rangle$. As this result is true for all states $|\phi\rangle$ we can write the unbiased evolution of the universe as

$$
|\Psi(\tau)\rangle=\left[U_{F}(\tau)+U_{B}(\tau)\right]\left|\psi_{0}\right\rangle .
$$

We call the process described by eq. (3) symmetric time evolution. This analysis can be repeated for an additional step of symmetric time evolution. After $N$ such steps the state of the universe is given by

$$
|\Psi(N \tau)\rangle=\left[U_{F}(\tau)+U_{B}(\tau)\right]^{N}\left|\psi_{0}\right\rangle .
$$

To simplify the description we have ignored the normalization of the state. Eq. (4) is represented as a binary tree in fig. $1(\mathrm{a})$.

(a)

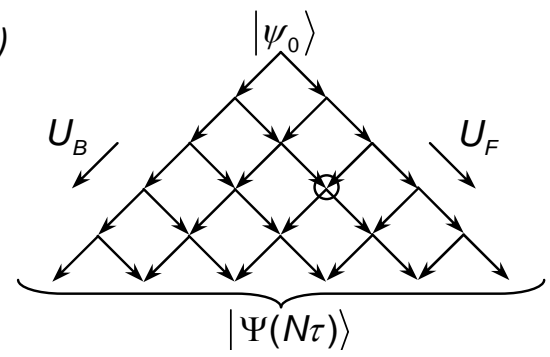

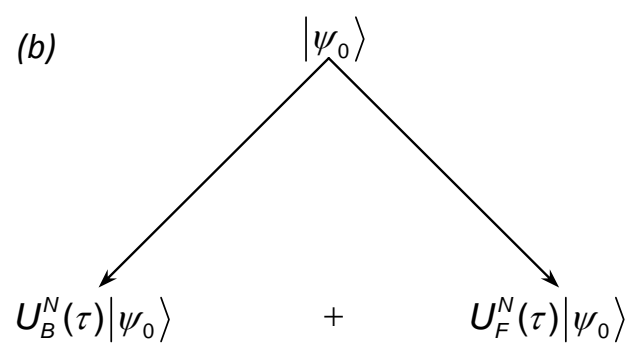

Figure 1: (a) and (b) are representations of eqs. (4) and (5), respectively, as binary trees. Leftward arrows

$(\checkmark)$ represent the application of $U_{B}$ and rightward arrows $(\checkmark)$ represent the application of $U_{F}$.

Expanding the $\mathrm{N}$-fold product on the right side of eq. (4) gives a series of $2^{N}$ terms each of which represents a different path through time. For example the terms $U_{F}^{N}(\tau)\left|\psi_{0}\right\rangle$ and $U_{F}^{N-1}(\tau) U_{B}(\tau)\left|\psi_{0}\right\rangle$ represent paths of $N$ steps containing zero and one backward step, respectively. The series can be separated into subseries whose terms involve the same number of steps in the forward and backward directions, but with steps in different orders. For example, the small circle in fig. 1(a) represents a subseries characterized by two forward steps and one backward step:

$$
U_{F}(\tau) U_{F}(\tau) U_{B}(\tau)\left|\psi_{0}\right\rangle+U_{F}(\tau) U_{B}(\tau) U_{F}(\tau)\left|\psi_{0}\right\rangle+U_{B}(\tau) U_{F}(\tau) U_{F}(\tau)\left|\psi_{0}\right\rangle .
$$

Note that the operators $U_{F}(\tau)$ and $U_{B}(\tau)$ do not commute if the Hamiltonian is not T invariant. In that case the terms of the subseries have been shown to interfere destructively (Vaccaro 2011). 
FFP12 2011

Udine 21-23 November 2011

In order to model the interference effect we use the conventional phenomenological model of neutral kaon decay (Lee 1973, Yao 2006) as the prototypical T violation process. We quantify the number of kaons as $f \times 10^{80}$ where $f$ is the fraction of the estimated $10^{80}$ total number of particles in the universe and set the size of the time step to the Planck time, i.e. $\tau \approx 10^{-44} \mathrm{~s}$. With these conditions, a relatively lengthy calculation (Vaccaro 2011) reveals that for $N \tau \gg f^{-1 / 2} 10^{-13} \mathrm{~s}$ eq. (4) becomes

$$
|\Psi(N \tau)\rangle \approx\left[U_{F}^{N}(\tau)+U_{B}^{N}(\tau)\right]\left|\psi_{0}\right\rangle .
$$

The right side comprises only two parts, one representing consistent evolution in the forward direction, $U_{F}^{N}(\tau)\left|\psi_{0}\right\rangle$, and the other representing consistent evolution in the backward direction, $U_{B}^{N}(\tau)\left|\psi_{0}\right\rangle$, as illustrated in fig. 1(b). Destructive interference has eliminated all other paths. This should be contrasted with the situation in eq. (4) for which the direction of the evolution can change from step to step as illustrated in fig. 1(a).

\section{New kind of irreversibility}

We now turn to new results. The derivation of eq. (4) relies on the construction of paths through time and it is this feature we wish to explore in more detail here. If the direction of time is known to be the forwards direction, say, then we can determine whether a universe that is in state $|A\rangle$ will be found in a different state $|B\rangle$ after a time interval $t$ from the probability

$$
\left|\left\langle B\left|U_{F}(t)\right| A\right\rangle\right|^{2} \text {. }
$$

A nonzero probability value means that there is a temporal path from $|A\rangle$ to $|B\rangle$. Expression (6) is essentially the probability of transitioning from $|A\rangle$ to $|B\rangle$ after a time delay of $t$.

In the case where the direction of time is not predetermined, we have seen in the previous section that the construction of temporal paths of $N$ steps each of duration $\tau$ is given by

$$
\left[U_{F}(\tau)+U_{B}(\tau)\right]^{N} \text {. }
$$

With this operator we can check whether a universe that is in state $|A\rangle$ can evolve to state $|B\rangle$ in $N$ time steps of unspecified direction by seeing if the (un-normalized) probability

$$
\begin{aligned}
P_{N}(B \mid A) & =\left|\left\langle B\left|\left[U_{F}(\tau)+U_{B}(\tau)\right]^{N}\right| A\right\rangle\right|^{2} \\
& =\left\langle A\left|\left[U_{F}^{\dagger}(\tau)+U_{B}^{\dagger}(\tau)\right]^{N}\right| B\right\rangle\left\langle B\left|\left[U_{F}(\tau)+U_{B}(\tau)\right]^{N}\right| A\right\rangle
\end{aligned}
$$

is nonzero. The converse situation where the universe can evolve from $|B\rangle$ to $|A\rangle$ is given by checking for a nonzero value of

$$
\begin{aligned}
P_{N}(A \mid B) & =\left|\left\langle A\left|\left[U_{F}(\tau)+U_{B}(\tau)\right]^{N}\right| B\right\rangle\right|^{2} \\
& =\left\langle B\left|\left[U_{F}^{\dagger}(\tau)+U_{B}^{\dagger}(\tau)\right]^{N}\right| A\right\rangle\left\langle A\left|\left[U_{F}(\tau)+U_{B}(\tau)\right]^{N}\right| B\right\rangle .
\end{aligned}
$$

For a non T-violating universe $H_{B}=H_{F}$ and so $U_{F}^{\dagger}(\tau)=\exp \left(i H_{F} \tau\right)=U_{B}(\tau)$ and $U_{B}^{\dagger}(\tau)=\exp \left(-i H_{B} \tau\right)$ $=U_{F}(\tau)$, and thus $P_{N}(B \mid A)=P_{N}(A \mid B)$. In other words, if a non T-violating universe can evolve from $|A\rangle$ to $|B\rangle$ it can also evolve from $|B\rangle$ to $|A\rangle$, as one would expect if both directions of time are allowed.

However, the situation is very different for a T violating universe, i.e. one for which for $H_{B} \neq H_{F}$. In this case the right sides of eqs. (7) and (8) are not equal in general. Indeed, in the extreme case, one may be zero while the other is not. To see this let $|B\rangle$ be given by

$$
|B\rangle=\left[U_{F}(\tau)+U_{B}(\tau)\right]^{M}|A\rangle
$$

where $M$ is an integer, for which we find 
FFP12 2011

Udine 21-23 November 2011

$$
\begin{aligned}
P_{N}(B \mid A) & =\left|\left\langle B\left|\left[U_{F}(\tau)+U_{B}(\tau)\right]^{N}\right| A\right\rangle\right|^{2} \\
& =\left|\left\langle A\left|\left[U_{F}^{\dagger}(\tau)+U_{B}^{\dagger}(\tau)\right]^{M}\left[U_{F}(\tau)+U_{B}(\tau)\right]^{N}\right| A\right\rangle\right|^{2} .
\end{aligned}
$$

In the special case where $N=M$

$$
P_{M}(B \mid A)=|\langle B \mid B\rangle|^{2}
$$

which is the square of the norm of $|B\rangle$. Normalizing the probability results in a value of unity. This implies that the evolution from $|A\rangle$ to $|B\rangle$ is guaranteed as would be expected from eq. (9). However for the converse case,

$$
\begin{aligned}
P_{N}(A \mid B) & =\left|\left\langle A\left|\left[U_{F}(\tau)+U_{B}(\tau)\right]^{N}\right| B\right\rangle\right|^{2} \\
& =\left|\left\langle A\left|\left[U_{F}(\tau)+U_{B}(\tau)\right]^{N+M}\right| A\right\rangle\right|^{2} .
\end{aligned}
$$

If the value of $M$ is sufficiently large that destructive interference eliminates all paths but two, as illustrated by eq. (5), then the state $|B\rangle$ can be written as

$$
|B\rangle \approx\left[U_{F}^{M}(\tau)+U_{B}^{M}(\tau)\right]|A\rangle
$$

and it follows that eq. (10) becomes

$$
P_{N}(A \mid B) \approx\left|\left\langle A\left|U_{F}^{N+M}(\tau)+U_{B}^{N+M}(\tau)\right| A\right\rangle\right|^{2}=\left|\left\langle A\left|U_{F}^{N+M}(\tau)\right| A\right\rangle+\left\langle A\left|U_{B}^{N+M}(\tau)\right| A\right\rangle\right|^{2} .
$$

Moreover, let the value of $M$ be such that

$$
\langle A \mid B\rangle=0
$$

and so $P_{0}(A \mid B)=0$. It would be reasonable to assume that the observable universe is not cyclical and never returns to the same state in a fixed direction of time. For our model this means that $\left\langle A\left|U_{F}^{N+M}(\tau)\right| A\right\rangle=0$ and $\left\langle A\left|U_{B}^{N+M}(\tau)\right| A\right\rangle=0$, and so from eq. (11)

$$
P_{N}(A \mid B) \approx 0
$$

for any positive value of $N$. We have succeeded in showing, therefore, that the universe does not evolve from $|B\rangle$ to $|A\rangle$ despite the fact that it is guaranteed to evolve from $|A\rangle$ to $|B\rangle$. This is a new kind of irreversibility. But to fully appreciate what it means we first need to consider the relationship between the two terms on the right side of eq. (5).

\section{Superposition of matter and antimatter}

If the state $\left|\psi_{0}\right\rangle$ is T invariant, i.e. if $\left|\psi_{0}\right\rangle=T\left|\psi_{0}\right\rangle$, the two components of the right side of Eq. (5) are related by the time reversal operation

$$
U_{B}(N \tau)\left|\psi_{0}\right\rangle=T U_{F}(N \tau)\left|\psi_{0}\right\rangle .
$$

Multiplying both sides by $C P$, where $C$ and $P$ are the charge conjugation and parity inversion operators, and invoking the CPT theorem shows that $U_{F}(N \tau)\left|\psi_{0}\right\rangle$ and $U_{B}(N \tau)\left|\psi_{0}\right\rangle$ are related by charge-parity conjugation. It is interesting to consider the case where the evolution $U_{F}(N \tau)\left|\psi_{0}\right\rangle$ symbolizes baryogenesis. In this case, if $U_{F}(N \tau)\left|\psi_{0}\right\rangle$ is dominated by matter then $U_{B}(N \tau)\left|\psi_{0}\right\rangle$ is dominated by antimatter. Every particle in $U_{F}(N \tau)\left|\psi_{0}\right\rangle$ has a corresponding partner antiparticle in $U_{B}(N \tau)\left|\psi_{0}\right\rangle$ and vise versa, and so a planet composed of matter in $U_{F}(N \tau)\left|\psi_{0}\right\rangle$ is correspondingly composed of antimatter in $U_{B}(N \tau)\left|\psi_{0}\right\rangle$. The two versions of the planet evolve independently of each other according to eq. (5) and so particle-antiparticle annihilation between the two versions of the planet would not occur. Moreover the evolution of the matter-dominated universe in $U_{F}(N \tau)\left|\psi_{0}\right\rangle$ is equivalent, by the CPT theorem, to the evolution of the antimatter-dominated universe in $U_{B}(N \tau)\left|\psi_{0}\right\rangle$. This symmetry between $U_{F}(N \tau)\left|\psi_{0}\right\rangle$ and $U_{B}(N \tau)\left|\psi_{0}\right\rangle$ implies that 
FFP12 2011

Udine 21-23 November 2011

evolution in the forward direction is equivalent to the evolution in the backward direction. The directions forward and backward are redundant in this sense. The two arrows in fig. 1(b) can therefore be considered to be one as illustrated in fig. 2 .

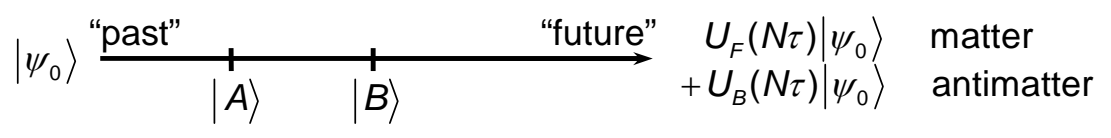

Figure 2. A reinterpretation of eq. (5) treating the two directions, forward and backward, as equivalent. The states $|A\rangle$ and $|B\rangle$ are related by the relationship given in eq. (9)

\section{Discussion}

We began with a model of the universe that has no presumed direction of time. Time evolution is described by a superposition of all possible paths that zigzag forwards and backwards through time as illustrated in fig. 1(a). We found that by incorporating sufficient T-violating processes only two paths representing always forwards and always backwards evolution survive destructive interference, as illustrated in fig. 1(b). We also showed that the two directions of time are essentially equivalent, as illustrated in fig. 2.

Moreover we found that the universe can evolve from one state $|A\rangle$ to another $|B\rangle$, as illustrated in fig. 2, but not vice versa. This endows the universe with a temporal direction pointing from a "past" to a "future". We have therefore described a physical mechanism which, if it exists in nature, could account for the directedness of time that we observe in our universe. This mechanism has the potential of being the cause of the direction of time. It is quite different to the asymmetry of the thermodynamic arrow which is an effect of a fixed direction of time and an incomplete knowledge of a system.

\section{References}

Christenson J H, Cronin J W, Fitch V L and Turlay R (1964) Evidence for the $2 \pi$ decay of the $K_{2}^{0}$ meson, Phys. Rev. Lett. 13, 138

Eddington A S (1928) The nature of the physical world, Macmillan, New York, USA

Feynman R P (1948) Space-time approach to non-relativistic quantum mechanics, Rev. Mod. Phys. 20, 367-387

Lee T D and Wolfenstein L (1965) Analysis of CP-noninvariant interactions and the $K_{1}^{0}, K_{2}^{0}$ system, Phys. Rev. 138, B1490-B1496

Price H (1996) Time's arrow and Archimedes' point, Oxford University Press, New York, USA

Vaccaro J A (2011) T violation and the unidirectionality of time, Found. Phys. 41, 1569-1596

Wigner E P (1959) Group theory and its application to the quantum mechanics of atomic spectra, Academic Press, New York, USA

Yao W -M et al. (2006) Review of particle physics, J. Phys. G: Nucl. Part. Phys. 33, 666-684 